\title{
Poxvirus-based vaccine platforms: getting at those hard-to-reach places
}
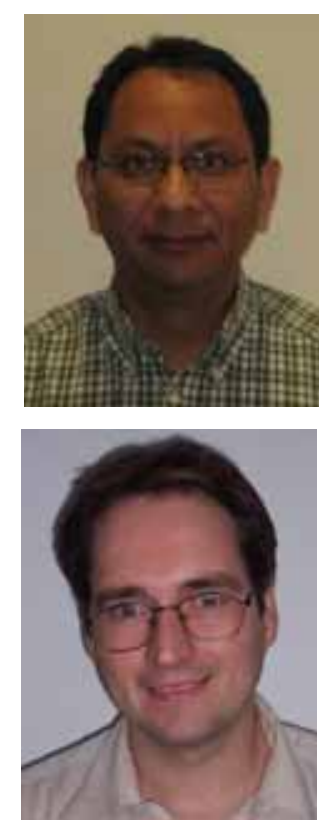

Rodolfo Nino-Fong \& James B Johnston ${ }^{\dagger}$

${ }^{\dagger}$ Author for correspondence Institute for N utrisciences \& $\mathrm{H}$ ealth, $\mathrm{N}$ ational Research Council Canada

550 U niversity Avenue, Charlottetown, $\mathrm{PE}$

C1A 4P3, Canada

Tel.: +1 9025668007 ;

Fax: +1 902367 7539;

jamesjohnston@nrc.ca

future, part of fso
'The prominence of poxvirusesas

candidate vaccine vectors

completes a circle begun in the

18th century when J enner first

tested the theory of vaccination

using Cowpox virus to inoculate

aga inst smallpox infection.'

The poxviruses are a largefamily of dsD N A viruses that includes some of the most notorious and extensively researched pathogens, most notably Variola virus, the causative agent of smallpox, and Vaccinia virus (VV), the prototypical poxvirus. Poxviruses are also notable among viruses for their large virion and genome sizes, the ability to replicate autonomous of host cell nuclear machinery, and the apparent absence of a virus-specific surface moiety for cell entry [1]. H istorically the subjects of extensive study because of their pathogenesis in humans and domestic animals, the eradication of smallpox as a human health concern in the 1980s greatly curtailed basic research involving poxviruses. In recent years, new avenues of biotherapeutic research, collectively known as virotherapeutics, have arisen to counter this trend, with the creation of numerous recombinant poxviruses for use as vaccine platforms, vectors for gene delivery and oncolytic agents in the treatment of cancer [2-4]. The prominence of poxviruses as candidate vaccine vectors completes a circle begun in the 18th century when Jenner first tested the theory of vaccination using Cowpox virus to inoculate against small lpox infection. Today, vaccination remains a critical intervention for preventing and limiting the spread of disease in the general population, as exemplified by the current campaign to vaccinate adolescent girls against human papilloma virus [5]. D espite many successes, effective vaccines for numerous prevalent conditions are lacking even as new infectious agents emerge and necessitate improved vaccination tools.

The capacity to genetically engineer poxviruses to alter pathogenicity and express heterologous genes encoding foreign antigens was being demonstrated even as small pox was being eradicated. This ability initiated the era of VV as a eukaryotic expression vector with broad potential as a vectorbased vaccine candidate. The properties of poxviruses that make them amenable for use as immunizing agents are well documented $[6,7]$. From a practical perspective, poxvirus vectors are stable in lyophilized form and are comparatively cheap and easy to manufacture. They have the potential to be safely administered by multiple pathways and elicit both mucosal and systemic responses. Compared with smaller viral vectors, the poxvirus genome can accommodate large foreign D N A inserts without disrupting viral stability, supporting multivalent delivery systems targeting several antigens from a single pathogen or antigens from several different pathogens. The lack of a restrictive surface receptor for entry also increases the range of hosts and cell types against which poxviruses may be applied. The principal flaw inherent in poxviral vectors is the issue of safety in the general population. The same adverse complications associated with the D ryvax ${ }^{\circledR}$ vaccine used in the smallpox campaign also apply to vaccine vectors based on live VV recombinants. Attempts to address this concern include attenuated VV strains, such as modified vaccinia Ankara (MVA) and NYVAC, with improved safety potential due to the loss of key pathogenic genes [8,9]. Avipoxvirus vectors based on Canarypox virus (CPV) and Fowlpox virus (FPV), such as ALVAC and TROVAC, are also extremely attractive from a biosafety standpoint $[10,11]$. Like other poxviruses, Avipoxviruses can gain entry to a wide variety of cell types but infection is aborted in nonavian cells. In addition, Avipoxvirus vectors do not cross-react with VV and elicit weaker vectordirected immune responses in mammals, supporting a multiple boosting regimen of treatment. Similarly, vectors derived from other poxvirus genera may also offer safer alternatives to VV [12,13].

\footnotetext{
'The principal flaw inherent in poxviral vectors is the issue of safety in the general population.'
}

Once their potential was recognized, numerous poxvirus vectors were engineered against diverse viral, bacterial and parasitic agents [14-16], as well as several cancerous states $[17,18]$. Theory 
is again becoming practice, as evidenced by human clinical trials [19-22] and the application of poxvirus-based vaccines in veterinary and agricultural sectors, especially in global rabies prevention programs [23]. Although there are currently no licensed vaccines for human parasitic diseases, VV vectors have been used to prevent cystic hydatid disease in sheep [24]. Protection against diverse animal viruses with economic implications has al so been demonstrated. Within the swine industry, recombinant poxviruses have been used to immunize against the devastating effects of porcine reproductive and respiratory syndrome virus [25] and pseudorabies [26]. Recently, avian influenza vaccines for chickens that use live FPV recombinants expressing strain-specific antigens have been approved for use in several countries [27], joining the list of FPV- and CPV-based veterinary vaccines that are commercially available [28].

Despite this progress, the 64 million dollar question remains - are poxviruses the $\mathrm{H}$ oly G rail in the fight against human diseases that have largely resisted concerted vaccine development efforts? To explore this question, we look at the role of poxvirus-based vaccines in two especially resistant conditions, malaria and HIV-AIDS. Despite overt dissimilarity in causative pathogens, both diseases are characterized by therapeutic stalemates in which interventions are able to reduce adverse effects but not eliminate the pathogen. The end goal in these conditions is not sterilizing immunity, but rather management of pathogen load during the critical early stages of infection in an effort to delay disease onset, reduce debilitating symptoms and limit transmission. To achieve this goal, vaccination strategies focus on restricting the spread of the pathogen as the host immune system reacts or boosting the initial responses to infection.

'Despite this progress, the 64 million dollar question rema ins are poxviruses the Holy Grail in the fight a gainst huma $n$ disea ses that have largely resisted concerted vaccine development efforts?'

The causative agent of malaria is a vector-borne, single-cell, eukaryotic parasite of the genus Plasmodium [29]. Vaccine development has been hampered by the complexity of the malarial parasite's life cycle, which incorporates several different replicative stages and forms. The parasite also exhibits the ability to replicate in terminally differentiated erythrocytes lacking $\mathrm{MHC}-\mathrm{I}$ expression, severely negating the impact of cellmediated immune responses. Thus, acquired immunity develops slowly as the host attempts to mount a response against a constantly evolving target without utilizing its primary resources against intracellular pathogens. 0 wing to this complexity, multivalent subunit vaccines are the vaccine strategy of choice [30], but efforts in this area have been limited by the need to identify protective rather than immunizing antigens and the continuing slow rate of host immunity acquisition. Candidate antigens are also problematic as a group in that they are poorly immunogenic and possess complex structures that challenge stability and purification.

'It can be argued that advances with recombinant poxvirus vector technologies continue to invigorate the next generation of vaccines, partic ula rly in such a reas as cancer immunothera py'.

Poxvirus-based vectors have the potential to alleviate many of these concerns. For example, poxvirus vectors have been shown to enhance the immunogenicity of recombinant protein-based vaccines, possibly through enhanced immune responses against the vector itself [31]. Poxviruses employ host protein production machinery to express the transgene product, thereby bypassing both the need for expensive production of purified protein and difficulties associated with retaining complex structures. Furthermore, the poxviral genome supports incorporation of multiple antigens from the different parasite forms within a single construct. This potential is best exemplified by NYVAC-Pf7, an attenuated VV-vector expressing seven different recombinant proteins, including the primary anti-invasive targets merozoite protein-1 and circumsporozoite protein (CSP) [32,33]. Clinical trials with NYVAC-pf7 demonstrated more effective immune responses than a comparable VV-based vector expressing only CSP and delayed onset of malaria symptoms, but did not provide complete protection. H owever, greater success with poxvirus vectors has been achieved using heterologous prime-boost regimens. For example, CD 8+ $\mathrm{T}$-cell responses capable of providing protection against sporozoite challenge were observed when a primary DNA vaccine against two antigens, CSP or trombospondinrelated adhesive protein (TRAP), was followed 
by a poxvirus-based booster [34]. This finding translated into similar reduced parasite loads in subsequent human trials using FPV and MVA expressing TRAP and several other antigens [35]. Recently, Phase II trials have begun and interest in the potential inherent in co-administration of poxvirus vectors and DNA vaccines as a new protocol of immunization continues [36].

Given the expense, poor accessibility and numerous adverse side effects associated with anti-H IV drug therapies, vaccines also represent the most cost-effective and practical control measure in HIV-AIDS. H owever, HIV and the malaria parasite share the ability to rapidly colonize host cells in a manner that dramatically reduces the host's ability to mount an immune response against the pathogen. Analogous to the multiple forms of the plasmodium parasite, the HIV virion is also constantly mutating in response to immune pressure and inherent genetic variability, creating a moving target for the host immune system. Early proof-of-principle studies in both human and nonhuman primates suggested great promise in inducing an immune response against H IV with VV recombinants expressing relevant viral envelope antigens [37,38], but quickly led to clinical trials that failed to deliver on the promise of efficacy. However, these trials did reveal that a primeboost regimen greatly improved the extent and duration of immune responses over virus-vectored antigen alone [39]. More recent studies using nonenvelope HIV antigens in combination with antiviral drugs or D N A vaccines have confirmed this finding and suggested the potential for therapeutic vaccination [40-42]. The scope and breadth of potential vaccine candidates developed and evaluated in the last few years are far too numerous for this editorial and have been reviewed elsewhere. However, among the more novel strategies being investigated is the incorporation of MVA-HIV envelope recombinants as orally available liposomal complexes. In mice, these complexes have been found to enhance envelope-specific cellular and humoral immune responses [43]. In addition, several cytokines, such as IL-15 and IFN - $\gamma$, have been incorporated into anti-H IV poxvirus vaccines in an attempt to promote $\mathrm{Th}$ 1-specific responses and induce memory [44,45].

Safety concerns, and the finding in the early HIV trials that pre-existing immunity to VV decreased the efficacy of VV-based vaccines for HIV [46], have focused recent efforts on the use of attenuated poxvirus species. Of note are the multisubunit MVA and NYVAC recombinants expressing HIV gag-pol-nef antigens in addition to the viral envelope genes that have been shown to consistently boost both $\mathrm{CD} 4^{+}$and CD 8 ${ }^{+} \mathrm{T}$-cell responses in HIV-1-infected patients $[47,48]$. In addition, novel inert poxvirus vectors have been developed in order to reduce the immune response against the vector itself, such as the highly attenuated VV substrain D airen-I [49] and VV strains lacking the B5 ectodomain against which many host immune responses are directed [50].

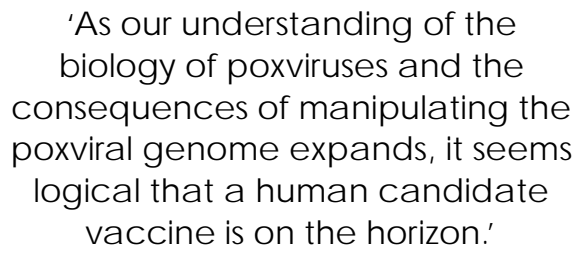

As described above, the poxvirus vector platform affords multiple vaccination strategies with diverse potential applications in human and animal disease. It can be argued that advances with recombinant poxvirus vector technologies continue to invigorate the next generation of vaccines, particularly in such areas as cancer immunotherapy [51]. The potential and excitement that accompanied the initial proposal in 1982 to use poxvirus-based vaccines has subsided to some extent, but the field continues to be one of extensive interest and research efforts. Successes within veterinary medicine have shown that the theory supporting the use of poxvirus vaccines is sound. As our understanding of the biology of poxviruses and the consequences of manipulating the poxviral genome expands, it seems logical that a human candidate vaccine is on the horizon. H owever, the biggest hurdle to overcome is likely never to be a scientific one. Rather, public perception and the fear of deliberately dosing oneself with a relative of an ancestral scourge will remain the greatest obstacle.

Financial \& competing interests disclosure

The authors have no relevant affiliations or financial involvement with any organization or entity with a financial interest in or financial conflict with the subject matter or materials discussed in the manuscript. This includes employment, consultancies, honoraria, sock ownership or options, expert testimony, grants or patents received or pending, or royalties.

No writing assistance was utilized in the production of this manuscript. 
Bibliography

1. M oss B: Poxviridae. In: The Virus and Their Replication. Knipe D M , H owley PM (Eds). Lippincott W illiams and W ilkins, Fields Virology, PA, USA 2849-2883 (2001).

2. M oroziewicz D, Kaufman H L: $G$ ene therapy with poxvirus vectors. Curr. 0 pin. M ol. Ther. 7, 317-325 (2005).

3. Arlen PM, Kaufman $\mathrm{HL}$, DiPaola RS: Pox viral vaccine approaches. Semin. 0 ncol. 32, 549-555 (2005).

4. Thorne $\mathrm{SH}, \mathrm{H}$ wang $\mathrm{TH}, \mathrm{Kirn} \mathrm{DH}$ : Vaccinia virus and oncolytic virotherapy of cancer. Curr. 0 pin. M ol. Ther. 7, 359-365 (2005).

5. Hakim AA, Lin PS, Wilczynski S, $N$ guyen $K$, Lynes $B$, Wakabayashi $M T$ : Indications and efficacy of the human papillomavirus vaccine. Curr. Treat. 0 ptions Oncol. 8(6), 393-401 (2008).

6. Boyle D B, Anderson M A, Amos R, Voysey R, Coupar BE: Construction of recombinant Fowlpox viruses carrying multiple vaccine antigens and immunomodulatory molecules. Biotechniques 37, 104-111 (2004).

7. Rocha $C D, C$ aetano $B C, M$ achado $A V$, Bruna-Romero 0 : Recombinant viruses as tools to induce protective cellular immunity against infectious diseases. Int. M icrobiol. 7, 83-94 (2004).

8. M eyer $H$, Sutter $G, M$ ayr $A$ : $M$ apping of deletions in the genome of the highly attenuated Vaccinia virus M VA and their influence on virulence. J. Gen. Virol. 72(Pt 5), 1031-1038 (1991).

9. Tartaglia J, Perkus M E, Taylor f et al.: N YVAC : a highly attenuated strain of Vaccinia virus. Virology 188, 217-232 (1992).

10. Plotkin $S A, C$ adoz $M$, M eignier $B$ et al.: The safety and use of canarypox vectored vaccines. D ev. Biol. Stand. 84, 165-170 (1995).

11. Skinner M A, Laidlaw SM, Eldaghayes I, Kaiser $P$, Cottingham $M G$ : Fowlpox virus as a recombinant vaccine vector for use in mammals and poultry. Expert Rev. Vaccines 4, 63-76 (2005).

12. Rziha $H J, H$ enkel $M$, Cottone $R, M$ eyer $M$, D ehio C, Buttner M : Parapoxviruses: potential alternative vectors for directing the immune response in permissive and nonpermissive hosts. J. Biotechnol. 73, 235-242 (1999).

13. Black D N : C apripoxvirus-based multivaccines. Dev. Biol. Stand. 84, 179-182 (1995).
14. Kreijtz JH, Suezer $Y$, van Amerongen $G$ et al.: Recombinant modified Vaccinia virus Ankara-based vaccine induces protective immunity in mice against infection with influenza virus H 5N 1. J. Infect. D is. 195, 1598-1606 (2007).

15. $M$ CShane $H$, Pathan $A A$, Sander $C R$ et al.: Recombinant modified Vaccinia virus Ankara expressing antigen $85 \mathrm{~A}$ boosts BCG-primed and naturally acquired antimycobacterial immunity in humans. $N$ at. M ed. 10, 1240-1244 (2004).

16. Miao J, Li X, Liu Z, Xue C, Bujard H, $C$ ui $L$ : Immune responses in mice induced by prime-boost schemes of the Plasmodium falciparum apical membrane antigen 1 (PfAM A1)-based D N A, protein and recombinant modified Vaccinia Ankara vaccines. Vaccine 24, 6187-6198 (2006).

17. Boursnell M E, Rutherford $E, H$ ickling JK et al.: C onstruction and characterisation of a recombinant Vaccinia virus expressing human papillomavirus proteins for immunotherapy of cervical cancer. Vaccine 14, 1485-1494 (1996).

18. Lindsey $K R$, Gritz $L$, Sherry $R$ et al.: Evaluation of prime/boost regimens using recombinant poxvirus/tyrosinase vaccines for the treatment of patients with metastatic melanoma. Clin. Cancer Res. 12, 2526-2537 (2006).

19. Arlen PM, Gulley JL: Therapeutic vaccines for prostate cancer: a review of clinical data. Curr. 0 pin. Investig. D rugs 6, 592-596 (2005)

20. M eyer RG, Britten CM , Siepmann U et al.: A Phase I vaccination study with tyrosinase in patients with stage II melanoma using recombinant modified Vaccinia virus Ankara (M VA-hTyr). Cancer Immunol. Immunother. 54, 453-467 (2005).

21. Bejon $P, M$ wacharo J, Kai $O$ et al.: A Phase $2 b$ randomised trial of the candidate malaria vaccines FP9 M E-TRAP and M VA M E-TRAP among children in Kenya. PL 0S Clin. Trials 1, e29 (2006).

22. Russell ND, Graham BS, Keefer M C et al.: Phase 2 study of an HIV-1 canarypox vaccine (VCP1452) alone and in combination with rgp120: negative results fail to trigger a Phase 3 correlates trial. J. Acquir. ImmuneD efic. Syndr. 44, 203-212 (2007)

23. Blancou J, Kieny M P, Lathe R et al.: $O$ ral vaccination of the fox against rabies using a live recombinant Vaccinia virus. N ature 322, 373-375 (1986).

24. $M$ arsland $B J$, Tisdall $D J, H$ eath $D D$, $M$ ercer AA: Construction of a recombinant orf virus that expresses an Echinococcus granulosus vaccine antigen from a novel genomic insertion site. Arch. Virol. 148, 555-562 (2003).

25. Shen $\mathrm{G}$, Jin $\mathrm{N}, \mathrm{M}$ a $\mathrm{M}$ et al.: Immune responses of pigs inoculated with a recombinant Fowlpox virus coexpressing G P5/G P3 of porcine reproductive and respiratory syndrome virus and swine IL-18. Vaccine 5, 4193-4202 (2007).

26. Fischer $\mathrm{T}$, Planz $\mathrm{O}$, Stitz L, Rziha H J: N ovel recombinant parapoxvirus vectors induce protective humoral and cellular immunity against lethal herpesvirus challenge infection in mice. J. Virol. 77, 9312-9323 (2003).

27. Bublot M , Pritchard N, Swayne DE et al.: D evelopment and use of fowlpox vectored vaccines for avian influenza. Ann. NY Acad. Sci. 1081, 193-201 (2006).

28. Poulet $H, M$ inke J, Pardo $M C$, Juillard $V$, N ordgren B, Audonnet J C: D evelopment and registration of recombinant veterinary vaccines. The example of the canarypox vector platform. Vaccine $25,5606-5612$ (2007).

29. Tuteja R: M alaria - an overview. FEBS J. 274, 4670-4679 (2007).

30. Ballou W R, Arevalo-H errera M, C arucci D et al.: U pdate on the clinical development of candidate malaria vaccines. Am. J. Trop. M ed. H yg. 71, 239-247 (2004).

31. Hutchings $\mathrm{CL}$, Gilbert $\mathrm{SC}, \mathrm{H}$ ill $\mathrm{AV}$, M oore AC: N ovel protein and poxvirusbased vaccine combinations for simultaneous induction of humoral and cell-mediated immunity. J. Immunol. 175, 599-606 (2005).

32. O ckenhouse CF, Sun PF, Lanar DE et al.: Phase I/II a safety, immunogenicity, and efficacy trial of NYVAC - Pf7, a poxvectored, multiantigen, multistage vaccine candidate for Plasmodium falciparum malaria. J. Infect. D is. 177, 1664-1673 (1998).

33. Tine JA, Lanar DE, Smith $D M$ et al.: NYVAC-Pf7: a poxvirus-vectored, multiantigen, multistage vaccine candidate for Plasmodium falciparum malaria. Infect. Immun. 64, 3833-3844 (1996).

34. Webster DP, DunachieS, Vuola JM et al.: Enhanced $T$ cell-mediated protection against malaria in human challenges by using the recombinant poxviruses FP9 and modified Vaccinia virus Ankara. Proc. N atl Acad. Sci. U SA 102, 4836-4841 (2005).

35. M cC onkey SJ, Reece W H, M oorthy VS et al.: Enhanced T-cell immunogenicity of plasmid D N A vaccines boosted by recombinant modified Vaccinia virus Ankara in humans. $N$ at. M ed. 9, 729-735 (2003). 
36. Weiss WR, Kumar $A$, Jiang $G$ et al.: Protection of rhesus monkeys by a D N A prime/poxvirus boost malaria vaccine depends on optimal DN A priming and inclusion of blood stage antigens. PLOS ONE 2, E1063 (2007).

37. Cho M W, Kim YB, Lee M K et al.: Polyvalent envelope glycoprotein vaccine elicits a broader neutralizing antibody response but is unable to provide sterilizing protection against heterologous Simian/human immunodeficiency virus infection in pigtailed macaques. J. Virol. 75, 2224-2234 (2001).

38. Cooney EL, Collier AC, Greenberg PD et al.: Safety of and immunological response to a recombinant Vaccinia virus vaccine expressing H IV envelope glycoprotein. Lancet 337, 567-572 (1991).

39. Evans TG, Keefer M C, Weinhold KJ et al.: A canarypox vaccine expressing multiple human immunodeficiency virus type 1 genes given alone or with rgp120 elicits broad and durable CD $8+$ cytotoxic $T$ lymphocyte responses in seronegative volunteers. J. Infect. D is. 180, 290-298 (1999).

40. Goonetilleke N, M oore S, D ally L et al.: Induction of multifunctional human immunodeficiency virus type 1 (H IV-1)-specific T cells capable of proliferation in healthy subjects by using a prime-boost regimen of DNA- and modified Vaccinia virus Ankara-vectored vaccines expressing H IV-1 G ag coupled to CD 8+T-cell epitopes. J. Virol. 80, 4717-4728 (2006).

41. Shinoda K, Xin KQ, KojimaY, Saha S, O kuda K, O kuda K: Robust HIV-specific immune responses were induced by $D N A$ vaccine prime followed by attenuated recombinant Vaccinia virus (LC $16 \mathrm{~m} 8$ strain) boost. Clin. Immunol. 119, 32-37 (2006).

42. H arrer $E$, Bauerle $M$, Ferstl $B$ et al.: Therapeutic vaccination of $\mathrm{HIV}$-1-infected patients on HAART with a recombinant H IV-1 nef-expressing M VA: safety, immunogenicity and influence on viral load during treatment interruption. Antivir. Ther. 10, 285-300 (2005).

43. N aito T, Kaneko Y, Kozbor D : O ral vaccination with modified Vaccinia virus Ankara attached covalently to TM PEG-modified cationic liposomes overcomes pre-existing poxvirus immunity from recombinant vaccinia immunization. J. Gen. Virol. 88, 61-70 (2007).

44. M cKee HJ, T'sao PY, Vera M, Fortes P, Strayer DS: D urable cytotoxic immune responses against gp120 elicited by recombinant SV 40 vectors encoding H IV-1 gp120 +/- IL-15. Genet. Vaccines Ther. 2, 10 (2004).

45. Giavedoni LD, Jones L, $G$ ardner M B et al.: Vaccinia virus recombinants expressing chimeric proteins of human immunodeficiency virus and $\gamma$ interferon are attenuated for nude mice. Proc. $\mathrm{N}$ atl Acad. Sci. USA 89, 3409-3413 (1992).

46. Belyakov IM, M oss B, Strober W, Berzofsky JA: M ucosal vaccination overcomes the barrier to recombinant vaccinia immunization caused by preexisting poxvirus immunity. Proc. Natl Acad. Sci. USA 96, 4512-4517 (1999).

47. H anke T, M cM ichael AJ, D orrell L: Clinical experience with plasmid D N Aand modified Vaccinia virus Ankaravectored human immunodeficiency virus type 1 clade $A$ vaccine focusing on T-cell induction. J. Gen. Virol. 88, 1-12 (2007).
48. Gomez CE, N ajera JL, Jimenez EP et al.: $\mathrm{H}$ ead-to-head comparison on the immunogenicity of two HIV/AIDS vaccine candidates based on the attenuated poxvirus strains M VA and N YVAC co-expressing in a single locus the H IV-1BX08 gp120 and H IV-1(IIIB) Gag-Pol-N ef proteins of clade B. Vaccine 25, 2863-2885 (2007).

49. O kamura T, Someya K, M atsuo K, H asegawa $A$, Yamamoto $N$, H onda $M$ : Recombinant vaccinia D Is expressing simian immunodeficiency virus gag and pol in mammalian cells inducesefficient cellular immunity as a safe immunodeficiency virus vaccine candidate. M icrobiol. Immunol. 50, 989-1000 (2006).

50. Viner KM , Girgis N, Kwak H, I saacs SN : $B 5$-deficient Vaccinia virus as a vaccine vector for the expression of a foreign antigen in vaccinia immune animals. Virology 361 , 356-363 (2007).

51. M oingeon P: Recombinant cancer vaccines based on viral vectors. D ev. Biol. (Basel) 116, 117-122 (2004).

Affiliations

- Rodolfo Nino-Fong Institute for Nutrisciences \& $\mathrm{H}$ ealth, $\mathrm{N}$ ational Research Council Canada, 550 U niversity Avenue, Charlottetown, PE, C1A 4P3, Canada

Tel.: +1 9025668007 ;

Fax: +1 902367 7539; rodolfo.nino-fong@nrc.ca

- JamesB Johnston Institute for N utrisciences \& $\mathrm{H}$ ealth, $N$ ational Research Council Canada, 550 U niversity Avenue, Charlottetown, PE, C1A 4P3, Canada

Tel.: +1 9025668007 ;

Fax: +1 9023677539 ; jamesjohnston@nrc.ca 